\title{
Interventional Laser Surgery for Oral Potentially Malignant Disorders - A Longitudinal Patient Cohort Study
}

\author{
P J Thomson ${ }^{1}$ \\ $M L$ Goodson ${ }^{1,2}$ \\ K Cocks ${ }^{3}$ \\ J E Turner ${ }^{4}$
}

${ }^{1}$ Oral \& Maxillofacial Surgery, School of Dentistry, University of Queensland, Oral Health Centre, Queensland 4006, Australia.

${ }^{2}$ Newcastle University Medicine Malaysia, 79200 Iskandar Puteri, Johor, Malaysia.

${ }^{3} \mathrm{KC}$ Stats Consultancy, www.kcstats.co.uk.

${ }^{4}$ Department for Health, University of Bath, BA2 7AY, UK.

e-mail: peter.thomson@uq.edu.au 


\begin{abstract}
Oral squamous cell carcinoma (OSCC) is a lethal disease, with rising incidence. In 2011, 6,767 new cases and 2,056 deaths occurred in the UK. Cancers are preceded by oral potentially malignant disorders (PMD), recognizable mucosal diseases harbouring increased, SCC risk offering clinicians a 'therapeutic window' to intervene. Contemporary practice remains unable to predict lesion behaviour or quantify malignant transformation (MT) risk. No clear management guidelines exist and the literature cannot determine whether early diagnosis and intervention prevents cancer. 773 laser treatments were performed between 1996 and 2014 in Newcastle Maxillofacial Surgery and efficacy of intervention examined by reviewing clinico-pathological details and clinical outcome for 590 PMD patients followed for a mean of 7.3yrs. Histopathology required 'up-grading' in 36\% on examining excision specimens. $74.2 \%$ of patients were disease free (DF), younger patients with 'lowgrade' dysplasia, whilst 9\% exhibited persistent disease and were older with proliferative verrucous leukoplakia (PVL). DF status was less likely for erythroleukoplakia $(p=0.022)$, 'high-grade' dysplasia $(p<0.0001)$ and with lichenoid inflammation $(\mathrm{P}=0.028)$. In $12 \%$, unexpected OSCC was identified, whilst $4.8 \%$ transformed to malignancy. Interventional laser surgery facilitates definitive diagnosis and treatment, allows early diagnosis of OSCC, identifies progressive disease and defines outcome categories. Evidence is lacking that intervention halts carcinogenesis. Multi-centre, prospective, RCTs are needed to confirm the efficacy of surgery.
\end{abstract}




\section{Introduction}

Oral squamous cell carcinoma (OSCC), probably the commonest head and neck malignancy, remains lethal and deforming due to local invasion, oro-facial destruction, metastasis to cervical lymph nodes and blood-borne tumour dissemination ${ }^{1}$. In 2011, 6,767 new cases and 2,056 deaths occurred in the UK whilst globally OSCC shows rising incidence with prognosis significantly compromised by advanced disease and late presentation? ${ }^{2}$.

The 'progression model' for oral carcinogenesis proposes that, following irreversible genotypic mutation, phenotypic epithelial disorganisation and dysmaturation changes occur which, if allowed to progress, lead to invasive OSCC $^{3}$. Such features preceding cancer are identifiable microscopically and collectively termed dysplasia ${ }^{4}$. In clinical practice, assessment of the degree of dysplastic change is made following incision biopsy and classification into mild, moderate or severe categories, according to the extent of atypical epithelium ${ }^{5}$.

It has been recognized for many years that a spectrum of distinct mucosal abnormalities, now termed potentially malignant disorders (PMD) and which accompany dysplasia, may be identified clinically, albeit non-specifically, during oral examination. There is, therefore, opportunity for early diagnosis and therapeutic intervention during this 'oral pre-cancer window'. PMD encompasses primarily localized lesions such as leukoplakia, erythroplakia and erythroleukoplakia, together with progressive multi-focal disorders such as proliferative verrucous leukoplakia $(\mathrm{PVL})^{7,8}$.

PMD identification in a patient does not mean inevitable malignant transformation (MT) will occur. Many lesions do not progress, while others spontaneously resolve, but it remains impossible in clinical practice to predict behaviour for individual cases. Nonetheless, PMD patients remain at increased risk of OSCC ${ }^{9}$.

'Potential malignancy' is difficult to define so it is unsurprising that previous treatment interventions have been non-specific with ill-defined goals and end-points; the few clinical trials in the literature concentrate on medical therapies and are compromised by small patient numbers and short study durations ${ }^{10}$. No study has demonstrated long-term lesion resolution, reduction in disease incidence or prevention of $\mathrm{MT}^{11}$. Arduino et $\mathrm{al}^{12}$ emphasized the need for long term patient studies to improve understanding of PMD natural history.

Lack of insight into PMD progression has resulted in a variety of proposed treatments based upon clinicians' preferences and experience. PMDs are mucosal lesions only so do not require the aggressive treatment necessary for removal or destruction of invasive cancer. It seems self-evident, therefore, to intervene early to excise dysplastic tissue at this 'pre-invasive' stage. 
Interventional laser therapies evolved following demonstrable failure of observational and medical treatments and limitations in conventional surgery to treat oral $P M D^{13,14,15,16,17}$. The use of carbon dioxide $\left(\mathrm{CO}_{2}\right)$ laser facilitates surgical management, providing precise excision, full histopathological lesion assessment, minimal post-operative morbidity and coordinated patient follow up ${ }^{14,17,18}$. Whilst excision is preferred, a limited role for ablation allows a defocused laser beam to destroy small or less dysplastic lesions particularly on gingiva / alveolar sites ${ }^{16}$.

Previous PMD treatment studies are limited by small patient numbers and varying follow up periods. Detailed, long term analysis of a large, well-defined PMD patient cohort undergoing coordinated treatment and follow-up was overdue.

\section{Aims of the Study}

In the absence of national PMD disease statistics or a robust evidence-base for treatment, the aims of this study were to profile patient demography and clinicopathological data for a patient cohort presenting to a specialist PMD service in NorthEast England, to determine clinical outcomes following interventional $\mathrm{CO}_{2}$ laser surgery and to assess treatment efficacy and its influence on the progress of oral carcinogenesis. 


\section{Method}

1. Caldicott Approval was obtained from Newcastle University / Newcastle upon Tyne Hospitals NHS Foundation Trust to facilitate anonymized, retrospective data collection from medical records, operating logs and pathology reports from PMD patients treated by the author and attending specialist services at Newcastle Dental and Royal Victoria Infirmary hospitals. Inclusion criteria required new, untreated single-site PMD confirmed by incision biopsy. Patients with previous or multi-focal PMD, and those with a history of OSCC or head and neck radiotherapy were excluded.

2. Patient and Treatment Details. Demographic and clinico-pathological details collected from patients undergoing $\mathrm{CO}_{2}$ laser surgery between August 1996 and December 2014 comprised: date of treatment, age and sex, clinical appearance and anatomical site of oral lesion, histopathology diagnoses for incision and post-laser excision biopsies, follow-up data and any further laser treatment. Clinical outcome was determined at the study census date (31 December 2014) unless malignancy supervened when this became study exit-point.

3. Histopathology Diagnoses. Incision biopsies were performed under the direction of the first author (PJT) and excision specimens obtained following interventional laser treatment as detailed previously ${ }^{14}$. Laser surgery was carried out by PJT, or by colleagues working under direct supervision, within 6 to 12 weeks following incision biopsy to avoid disease progression. Formalin-fixed tissue specimens were assessed via standardized histopathology examination by oral pathologists at the Royal Victoria Infirmary working to agreed diagnostic criteria with peer review and consensus grading ${ }^{4}$. Using the World Health Organization (WHO) classification, specimens were graded as mild, moderate or severe dysplasia, carcinoma-in-situ (CiS) or OSCC. In addition, presence of hyperkeratosis, lichenoid inflammation (LI), or diagnoses of PVL and chronic hyperplastic candidosis $(\mathrm{CHC})$ were recorded.

4. Clinical Outcome Categories. By reviewing case records, the authors assigned patients to one of the following clinical outcome categories: Disease Free (DF), the absence of PMD, Further Disease achieving DF status following additional intervention (Further/DF), Further Disease persisting despite intervention (Further/Persistent) and MT, if OSCC was confirmed by histopathological examination.

5. Statistical Analyses. Descriptive Statistics were used to summarise details of patient demography, clinical features and pathological diagnoses, together with documentation of treatment interventions, clinical outcome and follow-up data. Histopathology diagnoses were treated as categorical variables and testing of agreement between incision biopsy and post-laser excision biopsy diagnoses carried out using weighted kappa statistics; a coefficient of 1 represented perfect agreement. 
Clinical Outcomes were stratified as DF or Persistent Disease (further PMD or MT); success rates and 95\% Confidence Intervals $(\mathrm{Cl})$ were calculated. Multivariate logistic regression was used to analyse factors potentially prognostic of DF outcome (age at first treatment, sex, lesion appearance, anatomical site and histopathology data). Relationships between factors were explored using chi-square tests for categorical variables, correlation coefficients or logistic regression to check for collinearity. Factors were first explored univariately and then a multivariate model built using a stepwise procedure until all variables were significant at the $10 \%$ level. Candidate variables for the initial model were those significant at the $20 \%$ level univariately. If collinearity was suspected only the most significant univariate relationships were included in the model. MT was analysed using univariate Cox regression to investigate clinico-pathological variables that might influence time to transformation. All statistical analyses were performed using SAS/STAT ${ }^{\circledR} 9.3$ software (SAS Institute Inc, Cary, USA). 


\section{Results}

Table 1 summarizes the study results.

1. Patient Demography. 590 PMD patients were treated during the 19-year period. Mean age at presentation was 59.7 years, with 347 males (59\%) and 243 females (41\%); 513 (87\%) were current or ex-smokers and 496 (84\%) regularly consumed alcohol.

2. Clinical Features. 468 lesions were leukoplakias, with erythroleukoplakia (99) and erythroplakia (23) less common. Floor of mouth and ventro-lateral tongue sites were involved in 358 cases.

3. Histopathology Features. Histopathological grading, based upon the most significant diagnosis obtained from either incision or laser excision biopsy showed 522 lesions (88.5\%) exhibited dysplasia or CiS. Incision and post-laser excision biopsies were available for direct comparison in 609 cases: in 307 (50\%) diagnoses agreed, although in 220 (36\%) excision specimens were 'up-graded' due to more severe dysplasia (121 or 20\%) or OSCC diagnosis (99 or 16\%). In 82 cases (14\%), excision specimens were less severe than incision samples. Whilst overall kappa agreement was deemed moderate at 0.45 (95\% Cl 0.40 to 0.51), specific agreement regarding OSCC diagnosis between incision and excision biopsies was only 0.25 (95\% Cl 0.15 to 0.35$)$.

4. Patient Management. The initial treatment modality was laser excision in 526 patients (89\%) and ablation in $64(11 \%)$. In total, 773 treatments were performed on 590 patients: $513(87 \%)$ underwent 1 laser surgery, whilst $77(13 \%)$ required repeat intervention. The mean number of treatments per patient was 1.2 (Standard Deviation 0.6 ), with a median of 1 (range 1 to 8).

5. Clinical Outcome. Mean follow-up was 7.3 years (SD 5.1 years), with a median of 6 years (range 0 to 19 years). 438 patients $(74.2 \%, 95 \% \mathrm{Cl} 70.7$ to $77.8 \%)$ were DF at study census date. 404 patients achieved this immediately following laser surgery, whilst an additional 34 required further laser intervention; 77 repeat treatments were provided with the mean number to achieve DF 2.26 (range of 2 to 4) and a mean time of 32.88 months (range 4 to 130.9 months). Out of 87 patients who required repeat treatment, 53 exhibited persistent PMD at census. MT was seen in 99 cases; in $71(12 \%)$, OSCC diagnosis was made unexpectedly following histopathological examination of laser excision specimens, whilst a further 28 (4.8\%) underwent MT during follow-up; median time to malignancy was 87.3 months (95\% Cl 59.9 to 149.2). Overall, 80 OSCCs were identified at the same site as their PMD precursor 
whilst 19 arose at new, distinct sites. Erythroleukoplakias were most likely to exhibit malignancy ( $p=0.0019$; Fisher's exact test).

6. Statistical Analyses. Univariate logistic regression analyses of clinico-pathological features potentially influencing DF status are summarised in Table 2. PVL did not meet the $20 \%$ criteria for inclusion in the multivariate model and anatomical site was sparse and highly correlated to a number of other factors therefore was also excluded. The final multivariate model contained lesion appearance $(p=0.022)$, dysplasia $(p<0.001)$ and the presence of $\mathrm{LI}(p=0.028)$; Table 3. Erythroleukoplakic lesions had significantly lower odds of achieving DF status compared with leukoplakia (OR $0.51,95 \% \mathrm{Cl} 0.31$ to 0.83 ), whilst the odds of DF status were significantly increased for lesions exhibiting mild compared with severe dysplasia (OR 2.25, 95\% Cl 1.27 to 3.98). The likelihood of DF status was reduced for lesions exhibiting LI features (OR 0.49, 95\% Cl 0.26 to 0.93). 28 patients underwent MT, with the median time to MT in the cohort 87.3 months (95\% CI 59.9 to 149.2); Figure 1. Univariate Cox regression analysis showed no significant influence of clinicopathological variables on MT time, but this could be due to the small number of events in the analysis.

\section{Discussion}

1. Introduction. This data set of 590 PMD patients, undergoing standardised and coordinated treatment by a single surgeon offers additional insight into the natural history of PMD in North-East England, albeit in a defined hospital population. The study provides detailed outcome data recorded over a follow-up period of 19 years (mean 7.3 years).

2. Patient Demographics. Demographics confirmed a median patient age of 60 years and, with $59 \%$ of cases male and $41 \%$ female, a $1.4: 1$ male to female ratio. No significant relationships between age/sex and PMD presentation or clinical outcome were characterised which is similar to previous analyses ${ }^{14,19,20}$. Most patients regularly smoked tobacco and drank alcohol confirming these as relevant aetiological factors in Northern England ${ }^{3}$.

3. Clinico-Pathological Features. The majority of PMD lesions were leukoplakias arising on the floor of mouth and ventro-lateral tongue, consistent with previous observations, probably due to underlying site predisposition to epithelial cell dysregulation and carcinogenic influence ${ }^{21,22}$. $88.5 \%$ of lesions in this study exhibited dysplasia or CiS, emphasizing the 'high-risk' nature of PMD disease in this population ${ }^{17}$. Re-grading of tissue specimens in a research-orientated approach was not undertaken as this would not have reflected the 'real-world' nature of the study ${ }^{23}$.

Whilst the prognostic significance of PMD lesions exhibiting LI (sometimes termed lichenoid dysplasias) has proved controversial ${ }^{5,8,24}$, this study has confirmed that 
persistent PMD disease is associated with lesions exhibiting LI. Previous clinical studies have observed isolated erythroleukoplakic lesions with LI arising at ventrolateral tongue sites to be at 'high risk' of $\mathrm{MT}^{25,26}$. Whilst oral lichenoid lesions have not been routinely followed up continuously, this may be advisable to monitor for early cancerous change ${ }^{8}$.

4. Laser Treatment. Whilst the use of $\mathrm{CO}_{2}$ laser as an effective, low-morbidity PMD treatment modality has been documented previously ${ }^{14,16,17,19,20}$, this study has facilitated analysis of laser treatment in the largest patient cohort reported in the literature $^{17,27}$. Advantages of laser include a relatively bloodless surgical field, improved intra-operative accuracy, reduced post-operative pain, limited scarring, reduced damage to adjacent tissues, low complication rates and the ability to repeat treatment ${ }^{14,16,17,18}$. Laser excisions were performed in $89 \%$ of patients distinguishing this study from previous publications favouring ablative techniques ${ }^{28}$, the latter disadvantaged by lack of definitive biopsy and repeat application required for disease resolution. It is notable that $87 \%$ of patients in this study required only 1 intervention.

5. Clinical Outcome. This long-term cohort study has facilitated detailed stratification of clinical outcome data, although it is difficult to compare these with other publications due to differing diagnostic and clinical decision making, varying treatment protocols and inconsistent terminology seen in other papers ${ }^{15,17,29} .75 \%$ of study patients were, however, DF at census date confirming a degree of treatment efficacy for laser intervention, pertinent because nearly $90 \%$ of treated lesions exhibited significant dysplasia. In terms of characterisation, DF patients were generally younger with mild or moderate dysplasia and no LI.

Long term clinic follow-up helped distinguish patients DF after further treatment (34 or 5.8\%) from persistent disease despite intervention (53 or 9\%). Patients in the Further/DF category were younger, developed new lesions at different sites, had less severe dysplasia and disease resolved after multiple treatments. In contradistinction, Further/Persistent patients were older, commonly had PVL lesions on gingiva, alveolus and labial mucosa and underwent ablation rather than excision. Traditionally, PVL diagnoses were made late during disease history following progressive spread, high recurrence and $\mathrm{MT}^{7,30}$. This study helps emphasise the importance of early PVL recognition in specialist PMD clinics, but recognises that ablative treatment intervention may have reduced efficacy.

6. Malignant Transformation. MT rates for PMDs vary worldwide with quoted values ranging between 0.1 to $40 \%$, and an overall rate estimated at $12 \%$, making it difficult to counsel individual patients ${ }^{15,16,17}$. This study distinguished between 71 cases $(12 \%)$ in which pre-existing OSCCs were discovered upon PMD laser excision and $28(4.8 \%)$ in which OSCC development occurred post-treatment (4.8\%). The significance of early OSCC diagnosis is emphasised because all 71 patients were 
definitively treated by their initial laser intervention. Overall, 80 OSCC cases were identified at the same-site as precursor lesions whilst 19 developed at new sites during follow-up confirming the need for long-term surveillance post-treatment. Recognisable clinico-pathological features associated with MT included an erythroleukoplakic appearance, severe dysplasia or CiS in initial biopsies and floor of mouth/ventro-lateral tongue sites of origin.

7. Study Limitations. This was not a prospective, randomised controlled trial, nor was it multi-centre, and it is recognised that clinician bias may have influenced both patient recruitment and treatment. Reliance on pre-existing clinical records can be problematic because detail and accuracy vary over time. It was disappointing in this study to find incomplete data regarding tobacco and alcohol use during follow-up as these confounding factors may influence outcome. We have, however, previously observed few changes in tobacco and alcohol use in Newcastle PMD patients following treatment ${ }^{19,20}$. Patient satisfaction and quality of life issues were not assessed in this study; these areas of research are important in modern healthcare provision and will form an integral part of future, prospective studies ${ }^{31}$.

In conclusion, this study has succeeded in providing data on the demographics and clinico-pathological profile of PMD disease in North-East England, and has detailed long-term clinical outcome following intervention. Optimal treatment requires consistent diagnoses, effective intervention and coordinated patient follow-up. Whilst these have been demonstrated in this study cohort, we have not demonstrated reduction in overall cancer risk, although the early identification and treatment of OSCC in $12 \%$ of cases probably justifies intervention alone. Whilst further analyses of cases with poor clinical outcome (persistent PMD and MT) are warranted, the next major phase of research requires prospective, multi-centre trialling, ideally internationally, to truly define the efficacy of interventional surgery.

\section{Acknowledgements}

The authors acknowledge the invaluable assistance through the years of colleagues in the Departments of Cellular Pathology, Medical Physics, Anaesthesia and PeriOperative Care at the Newcastle upon Tyne Hospitals NHS Foundation Trust, without whom this clinical work and study would not have been possible.

\section{Declarations}

Funding: None

Competing Interests: None 
Ethical Approval: Newcastle University / Newcastle upon Tyne Hopsitals NHS Foundation Trust Caldicott Guardian Approval for Anonymised Patient Data Collection \& Retrospective Review of Hospital records ID 4143 (2015)

Patient Consent: Not required

\section{References}

1. Mehanna H, Paleri V, West CML, Nutting C. Head and neck cancer - Part 1: Epidemiology, presentation, and prevention. Br Medical Journal 2010341 : 663666.

2. Conway DI, Petticrew M, Marlborough H, Berthiller J, Hashibe M, Macpherson LMD. Socioeconomic inequalities and oral cancer risk: A systematic review and meta-analysis of case-control studies. Int J Cancer 2008122 : 2811-2819.

3. Thomson PJ. Oral Carcinogenesis. In: PJ Thomson (ed) Oral Precancer Diagnosis and Management of Potentially Malignant Disorders. Chichester: WileyBlackwell; 2012. p31-47.

4. Sloan P. Pathological aspects of oral precancer. In P J Thomson (Ed) Oral Precancer - Diagnosis and Management of Potentially Malignant Disorders. Chichester: Wiley-Blackwell; 2012. p93-106.

5. Speight PM. Update on oral epithelial dysplasia and progression to cancer. Head and Neck Pathol 20071 : 61-66.

6. Thomson PJ. Introduction. In: PJ Thomson (ed) Oral Precancer-Diagnosis and Management of Potentially Malignant Disorders. Chichester: Wiley-Blackwell; 2012. p1-12.

7. van der Waal I. Oral lichen planus and oral lichenoid lesions: a critical appraisal with emphasis on the diagnostic aspects. Med Oral Patol Oral Cir Buccal 200914 : e310-314.

8. Goodson $\mathrm{ML}$, Sloan $\mathrm{P}$, Robinson $\mathrm{CM}$, Thomson $\mathrm{PJ}$. Oral precursor lesions and malignant transformation - who, where, what and when? British Journal of Oral \& Maxillofacial Surgery 2015 53: 831-835.

9. Warnakulasuriya S. Global epidemiology of oral and oropharyngeal cancer. Oral Oncology $200945: 309-316$. 
10. Lodi G, Porter S. Management of potentially malignant disorders: evidence and critique. J Oral Pathol Med 200837 : 63-69.

11. Ribeiro AS, Salles PR, daSilva TA, Mesquita RA. A review of the nonsurgical treatment of oral leukoplakia. International Journal of Dentistry 2010 doi:10.1155/2010/186018.

12. Arduino PG, Bagan J, El-Naggar AK, Carrozzo M. Urban legends series: oral leukoplakia. Oral Diseases 201319 : 642-659.

13. van der Waal I. Potentially malignant disorders of the oral and oropharyngeal mucosa; terminology, classification and present concepts of management. Oral Oncology 200945 : 317-323.

14. Thomson PJ, Wylie J. Interventional laser surgery: an effective surgical and diagnostic tool in oral precancer management. International Journal of Oral \& Maxillofacial Surgery 200231 :145-153.

15. Mehanna HM, Rattay T, Smith J, McConkey CC. Treatment and follow-up of oral dysplasia - a systematic review and meta-analysis. Head \& Neck 200931 : 16001609.

16. Thomson PJ. Management of Oral Precancer. In: PJ Thomson (ed) Oral Precancer - Diagnosis and Management of Potentially Malignant Disorders. Chichester: Wiley-Blackwell; 2012. p107-137.

17. Thomson PJ. The role of interventional surgery in oral potentially malignant disorders. Faculty Dental Journal 2014 5: 84-89.

18. Goodson ML, Sugden K, Kometa S, Thomson PJ. Complications following interventional laser surgery for oral cancer and precancerous lesions. British Journal of Oral \& Maxillofacial Surgery $201250:$ 597-600.

19. Hamadah O, Thomson PJ. Factors affecting carbon dioxide laser treatment for oral precancer: A patient cohort study. Lasers in Surgery and Medicine 200941 :17-25.

20. Diajil AR, Robinson CM, Sloan P, Thomson PJ. Clinical outcome following oral potentially malignant disorder treatment: a 100 patient cohort study. International Journal of Dentistry 2013 Article ID 809248, 8 pages http://dx.doi.org/10.1155/2013/809248.

21. Thomson PJ, Potten CS, Appleton DR. Mapping dynamic epithelial cell proliferative activity within the oral cavity of man: a new insight into carcinogenesis? British Journal of Oral \& Maxillofacial Surgery 199937 : 377-383. 
22. Thomson PJ, Goodson ML, Booth C, Cragg N, Hamadah O. Predicting recurrence after treatment of oral precancer: Use of cell cycle analysis. British Journal of Oral and Maxillofacial Surgery 200846 : 370-375.

23. Dost F, LeCao K, Ford PJ, Ades C, Farah CS. Malignant transformation of oral epithelial dysplasia: a real world evaluation of histopathologic grading. Oral Surg Oral Med Oral Pathol Oral Radiol 2014117 : 343-352.

24. Patil S, Rao R, Sanketh S, Warnakulasuriya S. Lichenoid dysplasia revisited evidence from a review of Indian archives. J Oral Pathol Med 201444 : 507-514.

25. Thomson PJ. Malignant Transformation and Oral Cancer Development. In: PJ Thomson (ed) Oral Precancer - Diagnosis and Management of Potentially Malignant Disorders. Chichester: Wiley-Blackwell; 2012. 156-169 ISBN 978-1-4443-3574-3

26. Dost F, LeCao K, Ford PJ, Ades C, Farah CS. Malignant transformation of oral epithelial dysplasia: a real world evaluation of histopathologic grading. Oral Surg Oral Med Oral Pathol Oral Radiol 2014117 : 343-352.

27. Jerjes W, Hamdoon Z, Hopper C. $\mathrm{CO}_{2}$ lasers in the management of potentially malignant and malignant oral disorders. Head \& Neck Oncology 20124 : 17-22.

28. Brouns ER, Baart JA, Karagozoglu KH, Aartman IH, Bloemena E, van der Waal I. Treatment results of $\mathrm{CO}_{2}$ laser vaporisation in a cohort of 35 patients with oral leukoplakia. Oral Diseases 201319 : 212-216.

29. Thomson PJ. Clinical Outcome. In: PJ Thomson (ed) Oral Precancer Diagnosis and Management of Potentially Malignant Disorders. Chichester: WileyBlackwell; 2012. 138-155 ISBN 978-1-4443-3574-3.

30. Cerero-Lapiedra R, Balade-Martinez D, Moreno-Lopez L-A, Esparza-GomezG, Bagan JV. Proliferative verrucous leukoplakia: a proposal for diagnostic criteria. Med Oral Patol Oral Cir Bucc 201015 : e839-e845.

31. Thomson PJ, McCaul J, Ridout F, Hutchison I. To treat...or not to treat? Clinician views on oral potentially malignant disorder management. British Journal of Oral \& Maxillofacial Surgery 2015 53: 1027-1031. 
Table 1: Patient Demographic, Clinico-Pathological and Outcome Data for Treated PMD Patients (Number $=590$ ).

\begin{tabular}{|c|c|}
\hline Age & \\
\hline Mean (SD) & $59.7(12.6)$ \\
\hline Median (range) & $60.0(23-94)$ \\
\hline Sex & No. (\%) \\
\hline Male & 347 (58.8\%) \\
\hline Female & $243(41.2 \%)$ \\
\hline Lesion & No. (\%) \\
\hline Leukoplakia & $468(79.3 \%)$ \\
\hline Erythroleukoplakia & $99(16.8 \%)$ \\
\hline Erythroplakia & $23(3.9 \%)$ \\
\hline Site & No. (\%) \\
\hline Floor Of Mouth & $172(29.2 \%)$ \\
\hline Lateral Tongue & $130(22.0 \%)$ \\
\hline Buccal Mucosa & $59(10.0 \%)$ \\
\hline Palate & $57(9.7 \%)$ \\
\hline Ventral Tongue & $56(9.5 \%)$ \\
\hline Labial Commissure & $29(4.9 \%)$ \\
\hline Fauces / Retromolar Region & $26(4.4 \%)$ \\
\hline Gingiva & $21(3.6 \%)$ \\
\hline Alveolus & $18(3.1 \%)$ \\
\hline Labial Mucosa & $11(1.9 \%)$ \\
\hline Dorsum of Tongue & $11(1.9 \%)$ \\
\hline Histopathology Diagnosis (Most Significant) & No. (\%) \\
\hline Hyperkeratosis & $8(1.4 \%)$ \\
\hline Hyperkeratosis + Lichenoid Inflammation (LI) & $28(4.8 \%)$ \\
\hline Chronic Hyperplastic Candidosis & $17(2.9)$ \\
\hline Proliferative Verrucous Leukoplakia (PVL) & $15(2.5 \%)$ \\
\hline Mild Dysplasia & $118(20.0 \%)$ \\
\hline Mild Dysplasia + LI & $28(4.7 \%)$ \\
\hline Mild Dysplasia + PVL & $40(6.8 \%)$ \\
\hline Moderate Dysplasia & $105(17.8 \%)$ \\
\hline
\end{tabular}




\begin{tabular}{|l|r|}
\hline Moderate Dysplasia + LI & $24(4.0 \%)$ \\
\hline Moderate Dysplasia + PVL & $15(2.5 \%)$ \\
\hline Severe Dysplasia & $99(16.8 \%)$ \\
\hline Severe Dysplasia + LI & $8(1.4 \%)$ \\
\hline Severe Dysplasia + PVL & $4(0.7 \%)$ \\
\hline Carcinoma-in-Situ & $81(13.7 \%)$ \\
\hline Duration of Follow up* (Years) & $7.3(5.1)$ \\
\hline Mean (SD) & $6(0-19)$ \\
\hline Median (Range) & $1.2(0.6)$ \\
\hline Treatment Intervention per Patient & $1(1-8)$ \\
\hline Mean (SD) & \\
\hline Median (Range) & $404(68.4 \%)$ \\
\hline Clinical Outcome & $34(5.8 \%)$ \\
\hline Disease Free & $53(9.0 \%)$ \\
\hline Further / Disease Free & $99(16.8 \%)$ \\
\hline Further / Persistent Disease & \\
\hline Malignant Transformation & \\
\hline
\end{tabular}

*Time from first treatment to $31^{\text {st }}$ Dec 2014 
Table 2: Univariate Analysis of Clinico-Pathological Factors Influencing Disease Free Status

\begin{tabular}{|l|c|}
\hline Factor & Logistic $\mathbf{p}$-value \\
\hline Site & 0.001 \\
\hline Age & 0.026 \\
\hline Sex & 0.112 \\
\hline Lesion & $<0.001$ \\
\hline Dysplasia & $<0.001$ \\
(None, Mild, Moderate vs Severe) & \\
\hline LI & 0.043 \\
\hline PVL & 0.289 \\
\hline
\end{tabular}

Table 3: Final Multivariate Model of Clinico-Pathological Factors Influencing Disease Free Status

\begin{tabular}{|c|c|c|c|c|c|}
\hline \multicolumn{2}{|l|}{ Effect } & \multirow{2}{*}{ 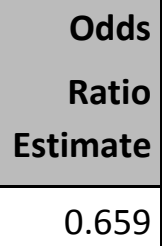 } & \multicolumn{2}{|c|}{$\begin{array}{c}95 \% \text { Wald } \\
\text { Confidence Limits }\end{array}$} & P-value \\
\hline Lesion & EK vs LK & & 0.269 & 1.619 & \multirow[t]{2}{*}{0.022} \\
\hline Lesion & ELK vs LK & $0.510 *$ & 0.314 & $0.828 *$ & \\
\hline Dysplasia & Mild vs Severe & $2.247^{*}$ & 1.270 & $3.976^{*}$ & \multirow[t]{3}{*}{$<0.0001$} \\
\hline Dysplasia & Moderate vs Severe & 1.693 & 0.954 & 3.006 & \\
\hline Dysplasia & None vs Severe & 0.628 & 0.369 & 1.068 & \\
\hline LI & No vs Yes & $0.489 *$ & 0.258 & 0.926 & 0.028 \\
\hline
\end{tabular}

*Significant at the $5 \%$ level 
Odds Ratio estimates $>1$ indicate increased likelihood of DF status, $<1$ decreased likelihood of DF status

(LK=Leukoplakia; ELK=Erythroleukoplakia; EK=Erythroplakia)

Figure 1: Kaplan-Meier Analysis plotting Time to Malignant Transformation (months) for PMD Precursor Lesions.

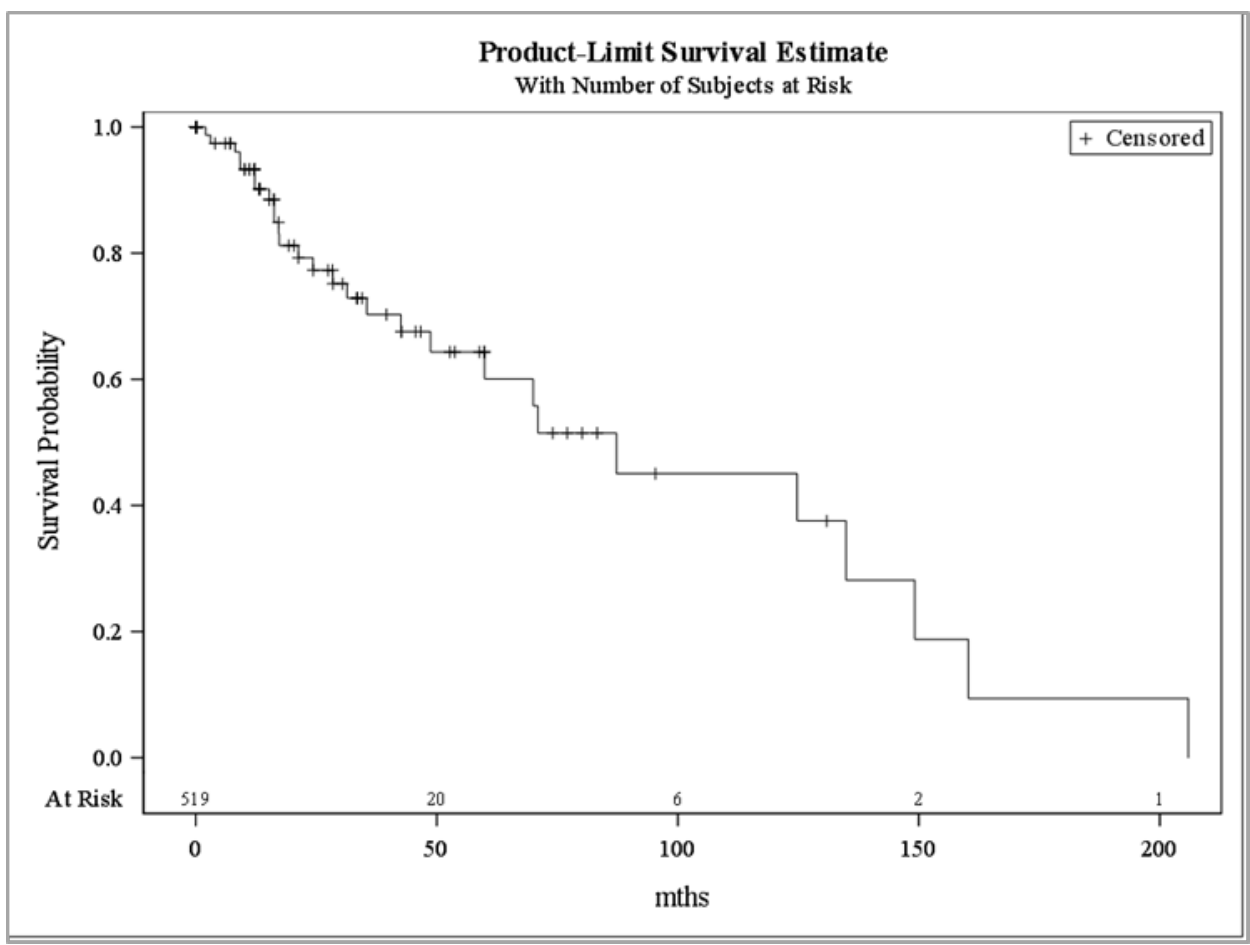

